\title{
Correction \\ Approximation by Superpositions of a Sigmoidal Function
}

\author{
G. Cybenko
}

In the paper "Approximation by Superpositions of a Sigmoidal Function" [C], the proof given for Lemma 1 is incorrect since it relies on the erroneous statement that simple functions are dense in $L^{\infty}(\mathbb{R})$. The author has pointed out that the proof in [C] can be corrected by changing, at the bottom of page 307 and the top of page 308 , the occurrences of $L^{\infty}(\mathbb{R})$ to $L^{\infty}(J)$ for a compact interval, $J$, containing $\left\{y^{\mathrm{T}} x \mid x \in I_{n}\right\}$, where $y$ is fixed. It should also be noted that the reduction of multidimensional density to one-dimensional density as in the proof of Lemma 1 had previously been obtained by Dahmen and Micchelli, using the same techniques, in work on ridge regression (see Lemma 3.2 of $[D M]$ ).

We thank Raymond T. Melton, who pointed out the error in the proof of Lemma 1 in [C] and supplied a proof, showing that the Fourier transform of the measure $\mu$ must be zero because the $\mu$-measure of every half-plane is zero [M].

\section{References}

[C] G. Cybenko, Approximation by superpositions of a sigmoidal function, Math. Control Signals Systems, 2 (1989), 303-314.

[DM] W. Dahmen and C. A. Micchelli, Some remarks on ridge functions, Approx. Theory Appl., 3 (1987), 139-142.

[M] R. T. Meiton, Comments on "Approximation by Superpositions of a Sigmoidal Function," personal communication, March 26, 1992. 